\title{
Calibration of a FDS-LC long counter for monitoring the neutron fluence at the HINEG facility
}

\author{
Bing Hong, Yanan Li, Mohamed Mazuga, Taosheng Li*,Yongfeng Wang, Xiang Ji, Chao Liu, Jieqiong Jiang, FDS Team ${ }^{1 *}$ \\ ${ }^{I}$ Institute of Nuclear Energy Safety Technology, Chinese Academy of sciences, \\ Key Laboratory of Neutronics and Radiation Safety, Hefei, Anhui, 230031, China \\ *Corresponding Author, E-mail: taosheng.li@fds.org.cn
}

\begin{abstract}
A FDS-LC long counter was developed to monitor the neutron fluence at the HINEG facility. The response function and the effective centre of long counter were calculated by using SuperMC code. The calibration of this long counter was performed in Research Facility, China institute of atomic energy. The results of experimental and simulative characterization of the FDS-LC long counter are persented in this paper. The comparison of the measured results with the calculated results shown a good agreement within $5 \%$.
\end{abstract}

\section{KEYWORDS: Long counter, Response, The effective centre, Calibration}

\section{Introduction}

The High Intensity D-T Fusion Generator (HINEG) with yield of $10^{12} \mathrm{n} / \mathrm{s}$ is constructed by institute of nuclear energy safety technology, chinese academy of science(INEST,CAS). It can be used for the neutronics, radiation technology, and other characteristic technology for advanced nuclear energy system-such as fusion/fission reactor and the calibration of measurement devices. In order to monitor the neutron fluence or the neutron emission at the HINEG facility, the neutron fluence monitoring system needs to be developed. The long counter is insensitive to $\gamma$-rays, high efficiency, and has a flat response function over a wide neutron energy range, which make it an ideal neutron detector for the measurements of parallel neutron fields ${ }^{(1)}$. Such a neutron detector is chosen to monitor the neutron fluence or neutron emission at the HINEG facility. In previous work, our team has designed a long counter having a relatively flat response from neutron energies $1 \mathrm{keV}$ to $20 \mathrm{MeV}$ by adding tungsten material and the maximal relative variation is $7.8 \%{ }^{(2)}$. The design is then further optimized by our team to extend its energy range from $1 \mathrm{keV}$ to $150 \mathrm{MeV}$ with response fluctuation less than $9 \%$. A long counter called FDS-LC based on this design is developed by our team considering actual technology at manufacturing process.

\section{Description of FDS-LC long counter}

The geometry of the FDS-LC long counter is based on the De Pangher long counter ${ }^{(3)}$. Figure 1 shows the structure of this long counter. This long counter is about $50.4 \mathrm{~cm}$ in diameter and $52.2 \mathrm{~cm}$ in length. It mainly consists of a $\mathrm{BF}_{3}$ thermal neutron counter, the inner and outer polyethylene, borated polyethylene absorption layer, chromium and lead metal $(\mathrm{n}, \mathrm{xn})$ converter. The outer polyethylene annulus and the borated-polyethylene are used to reduce the effect of scattered neutron. The $\mathrm{BF}_{3}$ proportional counter, manufactured by LND.INC, is installed in the centre of the cylinder of inner polyethylene. The external diameter of this proportional counter is $50.8 \mathrm{~mm}$, the nominal active is 215.7 $\mathrm{mm}$ and the pressure of the $\mathrm{BF}_{3}$ gas is about 150 torr. The cadmium sheet in the front face of long counter is used to absorb thermal neutron of incident direction. The polyethylene cylinder of $25 \mathrm{~mm}$ long is placed in front the $\mathrm{BF}_{3}$ counter to flatten the response of the low energy neutron.To improve the response in the intermediate neutron energies, the air annulus with $12 \mathrm{~cm}$ depth and $0.4 \mathrm{~cm}$ width is added on the inner cylinder moderator. To improve the response function at higher neutron energies, a batch of 12 of the chromium bar of dimension $1.7 \mathrm{~cm}$ diameter and $31 \mathrm{~cm}$ length are inserted in inner polyethylene, and a thick $0.5 \mathrm{~cm}$ lead layer is placed in outside of the $\mathrm{BF}_{3}$ tube.

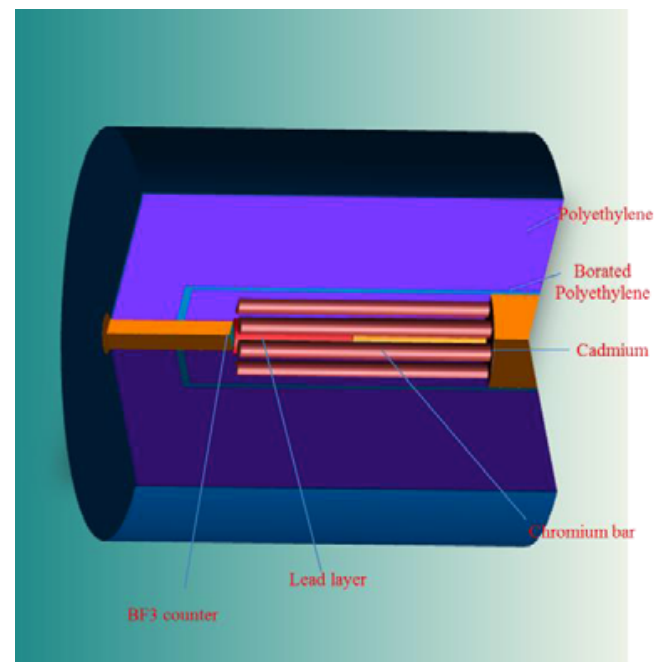

Figure 1. the schematic diagram of FDS-LC long counter 


\section{The characteristics of FDS-LC}

According to the actual geometric structure and material parameters of FDS-LC long counter, the long counter was modeled using the Super Monte Carlo (SuperMC) code ${ }^{(4)}$. The response function and the effective centre of FDS-LC long counter were calculated by using SuperMC code. The response function of FDS-LC long counter was calculated for the radioactive source $\left({ }^{241} \mathrm{Am}-\mathrm{Be},{ }^{252} \mathrm{Cf}\right)$ and for about hundreds of monoenergetic neutron in the range $1 \mathrm{keV}$ to $150 \mathrm{MeV}$. The calculated results revealed that the responses were almost flat from $1 \mathrm{keV}$ to $150 \mathrm{MeV}$ with the mean value $1.1 \mathrm{~cm}^{2}$. The effective centre of FDS-LC long counter was also calculated. The relationship between the effective centre of FDS-LC long counter and the neutron energy in the range $0.1 \mathrm{MeV}$ to $20 \mathrm{MeV}$ was also estimated.

\section{The Calibration of FDS-LC}

The FDS-LC long counter developed in this study was shown in Figure 2. It was calibrated using ${ }^{241} \mathrm{Am}-\mathrm{Be}$ source and $\mathrm{T}(\mathrm{d}, \mathrm{n})^{3} \mathrm{He}$ source in Research Facility, China institute of atomic energy. The FDS-LC long counter signal was acquired by the experimental acquisition system. The acquisition system consisted of an amplifier, a discriminator and a counter. The discriminator was used to discriminate between the neutron-induced signal and the $\gamma$-induced signal. The experimental results shown that the response of FDS-LC long counter was $1.11 \mathrm{~cm}^{2}$ for the $\mathrm{T}(\mathrm{d}, \mathrm{n})^{3} \mathrm{He}$ source and 1.06 $\mathrm{cm}^{2}$ for the ${ }^{241} \mathrm{Am}-\mathrm{Be}$ source, respectively. The calculated and measured results were also shown in Table 1.

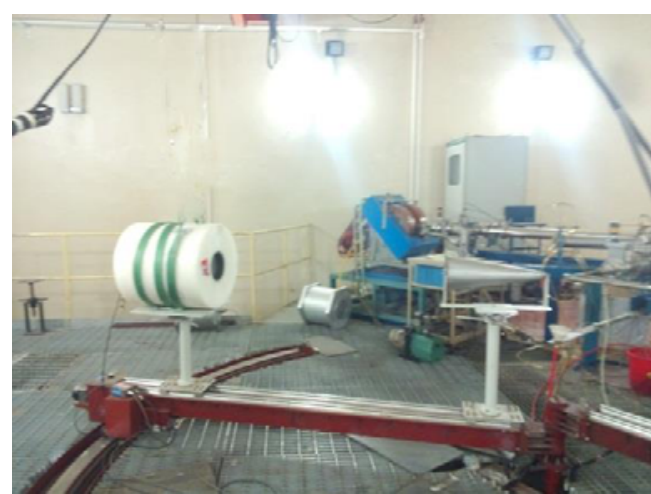

Figure 2. the calibration of FDS-LC long counter in Research Facility, China institute of atomic energy

\begin{tabular}{cccc}
\hline Source & $\begin{array}{r}\text { Energy } \\
(\mathrm{MeV})\end{array}$ & $\begin{array}{c}\text { Calculated } \\
\text { efficiency } \\
\left(\mathrm{cm}^{2)}\right.\end{array}$ & $\begin{array}{c}\text { Measured } \\
\text { Efficiency } \\
\left(\mathrm{cm}^{2}\right)\end{array}$ \\
\hline${ }^{241} \mathrm{Am}-\mathrm{Be}$ & 4.16 & 1.01 & 1.06 \\
$\mathrm{~T}(\mathrm{~d}, \mathrm{n})^{3} \mathrm{He}$ & 14.8 & 1.11 & 1.11 \\
\hline
\end{tabular}

Table 1 .Comparison of the calculated and measured effeciency for $241 \mathrm{Am}-\mathrm{Be}$ source and $\mathrm{T}(\mathrm{d}, \mathrm{n}) 3 \mathrm{He}$ source

\section{Conclusion}

The good agreement within 5\% was confirmed between the measured and calculated response for the ${ }^{241} \mathrm{Am}-\mathrm{Be}$ source and $\mathrm{T}(\mathrm{d}, \mathrm{n})^{3} \mathrm{He}$ source, when uncertainties with $\mathrm{k}=2$ are $5 \%$ for ${ }^{241} \mathrm{Am}-\mathrm{Be}$ source and $7 \%$ for $\mathrm{T}(\mathrm{d}, \mathrm{n})^{3} \mathrm{He}$ source, respectively. The results demonstrated that the design of the
FDS-LC long counter was valid and consistent with the design requirements. It is now available for monitoring the neutron fluence at the HINEG facility

\section{Acknowledgment}

This work was supported by the insititute of nuclear safety and technology, Chinese Academy of Scineces. And Thank Members of FDS team for their helpful advice.

\section{References}

1) H.Tagziria and D.J.Thomas, "Calibration and Monte Carto modelling of neutron long counters,"Nucl.Instruments Methods Phys.Res.Set. A Accel.Spectrometers.Detect.Assoc.Equip., vol.452,no. 3,pp.470-483,2000

2) Yanan Li,Taosheng Li,Gang Song ,et al. Response improved for neutron long counter.Radiation Protection Dosimetry.2015,164:93-96.

3) De Pangher, J., Nichols L L. "precision long counter for measuring fast neutron flux density.," $B N W L-260,1966$.

4) L.Song,J.,Sun,G.,Chen,Z.,Zheng,H. and Hu," Benchmarking of CAD-based SuperMC with ITER benchmark model," Fusion Eng.Des,vol.89.pp.2499-2503.2014. 